\title{
Dilated cisternae chyli. A sign of uncompensated cirrhosis at MR imaging
}

\author{
Sachit K. Verma \\ Thomas Jefferson University \\ Donald G. Mitchell \\ Thomas Jefferson University \\ Diane Bergin \\ Thomas Jefferson University \\ Yulia Lakhman \\ Thomas Jefferson University
}

AmyAthsting additional works at: https://jdc.jefferson.edu/radiologyfp Thomas Jefferson University

Part of the Radiology Commons

\section{$\frac{\text { Let us know how access to this document benefits you }}{\text { See next page for additional authors }}$}

\section{Recommended Citation}

Verma, Sachit K.; Mitchell, Donald G.; Bergin, Diane; Lakhman, Yulia; Austin, Amy; Verma,

Manisha; Assis, David; Herrine, Steven K.; and Parker, Laurence, "Dilated cisternae chyli. A sign of uncompensated cirrhosis at MR imaging" (2008). Department of Radiology Faculty Papers.

\section{Paper 4.}

https://jdc.jefferson.edu/radiologyfp/4

This Article is brought to you for free and open access by the Jefferson Digital Commons. The Jefferson Digital Commons is a service of Thomas Jefferson University's Center for Teaching and Learning (CTL). The Commons is a showcase for Jefferson books and journals, peer-reviewed scholarly publications, unique historical collections from the University archives, and teaching tools. The Jefferson Digital Commons allows researchers and interested readers anywhere in the world to learn about and keep up to date with Jefferson scholarship. This article has been accepted for inclusion in Department of Radiology Faculty Papers by an authorized administrator of the Jefferson Digital Commons. For more information, please contact: JeffersonDigitalCommons@jefferson.edu. 


\section{Authors}

Sachit K. Verma, Donald G. Mitchell, Diane Bergin, Yulia Lakhman, Amy Austin, Manisha Verma, David Assis, Steven K. Herrine, and Laurence Parker 


\section{Dilated cisternae chyli: a sign of}

\section{uncompensated cirrhosis at MR imaging}

Sachit K. Verma, ${ }^{1}$ Donald G. Mitchell, Diane Bergin, ${ }^{1}$ Yulia Lakhman, ${ }^{1}$ Amy Austin, ${ }^{1}$ Manisha Verma, ${ }^{2}$ David Assis, ${ }^{2}$ Stevene K. Herrine, ${ }^{2}$ Laurence Parker

${ }^{1}$ Department of Radiology, Thomas Jefferson University Hospital, Philadelphia, PA 19107, USA

2Department of Medicine, Thomas Jefferson University Hospital, Philadelphia, PA, USA

Correspondence to: Sachit K. Verma; email: medskv@yahoo.com

\section{Abstract}

Background: In order to retrospectively determine the frequency of dilated cisterna chyli (CC) on MR images in patients with cirrhosis, and to assess its value as a simple diagnostic imaging sign of uncompensated cirrhosis.

Methods: Study population included 257 patients (149 with pathologically proved cirrhosis and 108 control subjects without the history of chronic liver diseases) who had 1.5 T MR imaging. Cirrhosis patients were divided into compensated and uncompensated groups. Three independent observers qualitatively evaluated the visibility of CC $2 \mathrm{~mm}$ or greater in transverse diameter, identified as a tubular structure with fluid signal intensity. CC diameters greater than $6 \mathrm{~mm}$ were defined as dilated. Statistical analysis was performed by Student t-test and interobserver 
agreement via intraclass correlation coefficient.

Results: CCs with diameter $2 \mathrm{~mm}$ or more were recorded in 113 of $149(76 \%)$ cirrhotic patients and 15 of $108(14 \%)$ control subjects $(P<0.001)$. Dilated CCs were significantly more frequent in uncompensated than compensated cirrhotic patients ( $54 \%$ vs $5 \%, P<0.001)$. The sensitivity, specificity, accuracy, and positive predictive value of dilated CC for uncompensated cirrhosis were $54 \%, 98 \%, 80 \%$, and $96 \%$, respectively.

Conclusions: Dilated CC can be used as a simple and specific sign complimentary to other findings of uncompensated cirrhosis.

Key words: Cirrhosis—Cisterna chyli-Dilated—Lymph—MRI 
About $80 \%$ of the lymph formed in the liver leaves via hilar lymphatics and enters the thoracic duct at or near the cisterna chyli (CC) [1]. In cirrhosis, there is an increased hepatic production of lymph, which may cause distension of the thin-walled lymphatic channels, particularly if there is increased resistance at the venolymphatic junction such as due to elevated systemic venous pressure [2]. Dilatation of porta hilar lymphatics and CC in patients with portal hypertension has been shown by lymphangiography, endoscopic ultrasound, and at autopsy [3-7].

The CC is commonly visible on MR images as high-signal intensity (SI) filled structure on heavily T2-weighted images, with delayed enhancement several minutes after the intravenous administration of gadolinium contrast agent [8]. In our current practice at MR imaging, we often noted an enlarged $\mathrm{CC}$ in patients with uncompensated cirrhosis. Thus the purpose of this study was to determine the frequency of dilated CC on MR images in patients with cirrhosis, and to assess its value as a simple diagnostic imaging sign of uncompensated cirrhosis.

Material and methods

Patients

From July 2005 through December 2006 at our institution, hepatic MR imaging reports, clinical MR imaging requests, and patient charts were searched by one author (S.K.V) to identify patients with cirrhosis and patients without chronic liver diseases. This retrospective review was conducted according to a protocol approved by the Institutional Review Board for retrospective review of radiology, clinical, and 
pathology records. Informed consent was not required. These cases were then cross-referenced to the histopathologic records to select patients with pathologically proved cirrhosis. Patients were excluded if they had a history of hepatic resection, cholecystectomy, or a malignant hepatic tumor larger than $2 \mathrm{~cm}$, as these may affect the morphology of cirrhotic liver. In patients having more than one MRI study, the first MRI study was used for initial analyses.

Consequently, this study population included a total of 257 consecutive patients, 149 with pathologically proved cirrhosis ( 82 men and 67 women; age range $22-87$ years; mean age 52.4 years) and 108 control subjects with no clinical evidence of chronic liver diseases (63 men and 45 women; age range 22-81 years; mean age 53.3 years). The presence of cirrhosis was documented histologically and/ or by MR features of cirrhosis, i.e., irregular, nodular liver surfaces with accompanying signs of portal hypertension such as the presence of ascites, esophageal varices, and/or splenomegaly. Among 149 cirrhotic patients, 43 patients had compensated cirrhosis and 106 patients had uncompensated cirrhosis based on the Child-Pugh classification (Child grades A, B, C) [9, 10]. Uncompensated cirrhosis was defined as the presence of at least two of the following five criteria: ascites, encephalopathy, hyperbilirubinemia $>3 \mathrm{mg} / \mathrm{dL}$, hypoalbuminemia $<3.2 \mathrm{~g} / \mathrm{dL}$, and prothrombin time $>3$ s [11].

Patients in the control group had undergone MR imaging for reasons other than chronic liver diseases (e.g., suspected liver cyst or hemangioma, benign disease of other organs). Reports indicated benign hepatic lesions including hemangioma, cyst, focal nodular hyperplasia in 59 , pancreatic and biliary system abnormality in 19 , liver 
metastasis in 11 , normal in 10 , or undetermined due to insufficient clinical data in 9 . Cirrhosis patients had been referred for MR imaging to evaluate the severity of cirrhosis and portal hypertension, to acquire preoperative studies before liver transplantation, or to evaluate suspected hepatocellular carcinoma.

One of the authors (S.K.V) retrospectively reviewed all pathology reports that were entered in the patient's clinical history. Cirrhosis was caused by viral infection (hepatitis B [ $n=25], C$ [ $=51]$, or $B$ and C [ $=19])$, alcohol abuse $(n=35)$, primary biliary cirrhosis $(n=10)$, a1-antitripsin deficiency $(n=4)$, or cryptogenic $(n=5)$. Cirrhosis was histopathologically confirmed by means of percutaneous liver biopsy in 124 patients, liver transplantation in 10, and transjugular liver biopsy in 15 . We were able to determine Child-Pugh classification from the available clinical records in 135 of 149 patients with cirrhosis. Forty-one were classified as having Child A, 18 patients as having Child B, and 76 patients as having Child C. Of 149 cirrhotic patients, 124 had only one MR examination and 25 had two or more MR examinations.

MR imaging

All MR examinations were performed during suspended respiration with a 1.5-T system (Signa; General Electric Medical Systems, Milwaukee, WI, USA; or Intera; Philips Medical Systems, Best, The Netherlands) and a 4-channel torso phased array coil. Coronal and transverse two-dimensional single-shot fast spin-echo (FSE) T2-weighted MR images (effective echo time, 180-200 ms), transverse fat suppressed FSE T2-weighted (2500-4000/80-90 [repetition time (ms)/echo time (ms)]), spoiled dual gradient-echo T1-weighted in-and out-of-phase (120-200/2.3 
and $4.6 \mathrm{~ms}, 90^{\circ}$ flip angle) MR images were obtained. Parameters for twodimensional images included a section thickness of $5-8 \mathrm{~mm}$ with an intersection gap 0-1 mm; a 256 × (160-192) matrix, a $32 \mathrm{~cm}$ transverse field of view and $24 \mathrm{~cm}$ anteroposterior field of view, one or fewer signals acquired. Three-dimensional (3D) dynamic enhanced spoiled gradient-echo dynamic MR images were obtained with 5 $\mathrm{mm}$ section thickness in $2.5 \mathrm{~mm}$ increments by using zero interpolation, 4-6/3-2.1 and $12-20^{\circ}$ flip angle parameters were otherwise similar to those of the twodimensional imaging. Twenty milliliters of gadopentate dimeglumine was administered intravenously by using a power-injector at $2 \mathrm{~mL} / \mathrm{s}$ followed by a $20-\mathrm{mL}$ saline flush. Imaging was initiated at a time intended to optimize first-pass arterial enhancement by using a timing bolus sequence, or observing the enhancement on images reconstructed in real-time. In all patients, 3D-gradient echo images were obtained during breath-hold before (non-enhanced phase), immediately after (hepatic arterial phase), and $30 \mathrm{~s}$ after (portal venous phase) the injection. Delayed phase (approximately after 3-5 min) images of the entire liver were obtained by using contiguous $5 \mathrm{~mm}$ thick fat suppressed two-dimensional single section technique (19/2, 30ำ flip angle), and by using 3D gradient-echo sequence described earlier. The acquisition of two-dimensional single section delayed phase images was included in our routine protocol because these images have less motion artifact and higher vascular SI as compared with 3D delayed phase images.

Review of records and images Images of subjects in the control and cirrhosis groups were presented to reviewers in random order. MR images of each patient were evaluated on a computer monitor 
on a picture archiving and communication system workstation (Canon Medical Systems, Irvine, CA, USA) retrospectively and independently by three radiologists (S.K.V., D.B., and Y.L with 3, 10, and 2 years, respectively, of body MRI experience) who were blinded to the final diagnosis with regard to the presence of cirrhosis, and they evaluated the images qualitatively for the presence of $\mathrm{CC}$. When there was disagreement in their opinions regarding the visibility of $\mathrm{CC}$, a majority opinion was used as the final decision. CC was considered visible if a ductal or saccular fluid structure in the prevertebral region below the diaphragm and SI similar to that cerebrospinal fluid was visualized in the retrocrural space usually to the immediate right of the abdominal aorta at the level of the L1-L2 vertebral body. In order to reduce subjective judgments involved with identifying and measuring structures with diameter less than 2 pixels, we restricted analysis to CCs with in plane diameter of 2 $\mathrm{mm}$ or greater. For objectivity and reproducibility of the image analysis performed in this study, the criteria to identify CC were provided on the basis of features observed on precontrast and dynamic phase images and of prior published descriptions of the appearance and enhance- ment characteristics of CC [1]. The radiologists defined a structure as CC if it showed high SI similar to spinal canal on heavily T2-weighted single shot FSE images and homogeneous enhancement on delayed phase images with no enhancement during the arterial and portal venous phases. Lymph nodes, which also enhance on delayed images, were distinguished from lymphatic channels due to their enhancement on early phase images, and their SI lower than fluid on heavily T2-weighted single shot FSE images. Images were reviewed on an independent imaging workstation. Each reviewer individually chose images for 
measurement on the basis of clarity of the landmarks. CCs with minimum transverse diameter of $2 \mathrm{~mm}$ constituted the study group. A cut-off point of $6 \mathrm{~mm}$ was used to define dilated CC [12]. Maximum transverse diameters at its widest part were recorded on axial heavily T2-weighted single shot FSE using electronic caliber. Presence or absence of ascites was noted. The growth rate of CC was expressed as diameter doubling time [13] for the available serial MRI follow-up.

Statistical evaluation

Statistical analysis was conducted using SAS V. 9.13 for Windows (2006; SAS Institute, Gary, NC, USA). Descriptive statistics (mean, SD) were provided where appropriate. Comparisons between the frequencies of dilated CC among compensated vs uncompensated cirrhotic subjects, and CC diameters on serial MRI, were conducted by using a two-tailed Student t-test. A P-value of less than 0.05 indicated a statistically significant difference. The sensitivity, specificity, accuracy, and positive predictive value of dilated CC in uncompensated group were also calculated.

Results

Cisterna chyli maximum transverse diameters were recorded in 113 of 149 (76\%) cirrhotic patients (mean $4.8 \mathrm{~mm}$; range $2-13 \mathrm{~mm}$ ) and in 15 of $108(14 \%)$ control subjects (mean $2.4 \mathrm{~mm}$; range $2-4 \mathrm{~mm})(P<0.001)$. Among the cirrhotic patients, CCs with maximum transverse diameter ranged from 2 to $11 \mathrm{~mm}$ (mean $2.7 \mathrm{~mm}$ ) in 25 of $43(58 \%)$ compensated and 2 to $13 \mathrm{~mm}$ (mean $5.4 \mathrm{~mm}$ ) in 88 of $106(83 \%)$ 
uncompensated cirrhosis patients $(P<0.001)$ (Table 1). Dilated CCs with maximum transverse diameter (range $6-13 \mathrm{~mm}$; mean $7 \mathrm{~mm}$ ) were seen in 2 of $43(5 \%)$ in compensated and 57 of $106(54 \%)$ in uncompensated cirrhosis patients $(P<0.001)$ (Fig. 1) (Table 1). None of the control patients had dilated CC. Between two patients with compensated cirrhosis with dilated CCs (diameters 11 and $6 \mathrm{~mm}$; mean 8.5 $\mathrm{mm}$ ), one had associated evidence of right-sided cardiac decompensation (Fig. 2) and the other had prior history of pancreatitis. The sensitivity, specificity, accuracy, and positive predictive value of dilated CC for uncompensated cirrhosis were $54 \%, 98 \%, 80 \%$, and $96 \%$, respectively. Among the 25 patients with serial MRI, the diameter of the $\mathrm{CC}$ on first MR images ranged from 3 to $11 \mathrm{~mm}$ (mean $5.6 \mathrm{~mm}$ ) and 4 to $12 \mathrm{~mm}$ (mean $6.6 \mathrm{~mm}$ ) on last MRI (mean follow-up 282 days) (not significantly different, $P=0.13$ ). Child-Pugh classification was $C$ in 23 of $25 ; 2$ other patients progressed from Child $B$ to Child $C$ based on the increase in the bilirubin and development of ascites. In these two patients, CC diameter on first MRI ranged from 4 to 5 (mean $4.5 \mathrm{~mm}$ ) and 5 to 6 (mean $6.6 \mathrm{~mm}$ ) on last MRI (mean follow-up 278 days) (Fig. 3). Interobserver reliability assessment Intraclass correlation coefficient (ICC) assessment as described by Shrout and Fleiss [14] indicated excellent to almost perfect agreement. ICC for CC measurement in compensated cirrhosis, uncompensated cirrhosis, and control subjects were $0.99,0.97$, and 0.98 . ICC for the measurement of dilated CC in compensated cirrhosis, uncompensated cirrhosis, and control subjects were 1.0, 0.97, and 0.97. 
Discussion

The results of the present study confirm the ability to identify CC by MRI in healthy volunteers and in patients with cirrhosis. Further, dilated CC had high specificity and positive predictive value for uncompensated cirrhosis, present in $54 \%$ of patients with uncompensated cirrhosis with excellent interobserver agreement.

Few previous studies have reported the imaging appearance of CC $[15,16]$; the frequency of seeing the CC on lymphangiography ranged from $30 \%$ to $53 \%$ [4], but only $1.7 \%$ on CT [17] On MRI, Erden et al. [18] detected CC in $96 \%$ of patients on heavily T2-weighted images. Unlike these investigators, we used a threshold diameter of $2 \mathrm{~mm}$ for identifying $\mathrm{CC}$ because of potential variability in identifying structures smaller than a pixel. This probably accounted for our lower rate of visible CC of $76 \%$ in cirrhotic patients and $14 \%$ of control subjects.

Lymphatics from the lower portion of the body converge in the lumbar region of the abdominal cavity to form the $\mathrm{CC}$, which extends for about $6 \mathrm{~cm}$ just to the right of the abdominal aorta. At the level of the 12th thoracic vertebra, the CC narrows and becomes the thoracic duct. In patients with portal hypertension, hepatic hilar lymphatics become distended and obstruct the hepatic venules. This phenomenon of increased lymph production in patients with advanced liver diseases is caused by disturbance in the drainage of vascular flow from the sinusoid to the central or terminal hepatic veins associated with lobular distortion $[7,19,20]$.

We speculate that in uncompensated cirrhosis any rise in pressure in the portal system will bring about an increase in liver and splanchnic lymph production, which 
is a factor responsible for CC dilatation [21, 22]. Little is known about the growth rate of such lymphatic channels [3]; however, in our study we did not find any significant change of diameter of CC among 25 patients over the period of less than 1 year.

It should be noted that the CC was visible in $14 \%$ of the control subjects. This may relate to the spectrum of normal physiology, but it is possible that some of these patients had increased thoracic duct lymph flow due to other conditions such as pancreatic and biliary obstruction, state of hydration (e.g., right-sided cardiac decompensation with elevated hepatic venous pressure) [23]. None of the control patients had CC wider than $6 \mathrm{~mm}$, in contrast to $5 \%$ of those with compensated cirrhosis and $54 \%$ with uncompensated cirrhosis. The $54 \%$ (57 of 106$)$ sensitivity of dilated CC for determining uncompensated cirrhosis is relatively low. However, its high specificity (98\%; 149 of 151$)$ renders this sign as complementary to other findings of uncompensated cirrhosis that may be more sensitive [24, 25].

We acknowledge the following limitations of our study. We did not compare MRI with another gold standard, but our interobserver agreement for detection rate of CC among the readers was quite high, with acceptable interobserver agreement. We were not able to confirm our imaging findings by conventional direct or indirect portographic methods in patients with portal hypertension. Conventional portography is invasive and therefore was not performed in most patients. We could not evaluate changes in visibility of the $\mathrm{CC}$ in relation to progression from compensated to uncompensated cirrhosis, since only short-term follow-up was possible. A further prospective study would be necessary to determine whether the visibility or diameter of $\mathrm{CC}$ has prognostic significance or can be used to monitor disease progression. 
Our control patients were chosen on the basis of imaging technique and period of imaging identical to that of the patients with cirrhosis. We did not match sex, race, or socioeconomic status between groups. However, we are not aware of any data to suggest that CC differs with respect to these variables. Since the control population was drawn from among people examined with MR imaging performed because they were suspected of having disease, this population may not be representative of the general population or of patients without cirrhosis who are at risk for cirrhosis. Another limitation was that the diameter of CC was determined on the basis of MR imaging, not by means of histological confirmation. Pathologic proof, however, would not be practical in the clinical setting.

In conclusion, although CC is commonly visible in subjects without liver disease or with clinically compensated cirrhosis, dilated CC found at MR is specific for uncompensated cirrhosis.

\section{References}

1. Lee KCY, Cassar-Pullicino VN (2000) Giant cisterna chyli: MRI depiction with gadolinium-DTPA enhancement. Clin Radiol 55:51- 55

2. Rusznyak I, Foldi M, Szabo G (1967). Lymphatics and Lymph Circulation 2nd edn Pergamon: Oxford, 197-598

3. Vignaux O, Gouya H, Dousset B, et al. (2002) Refractory chylothorax in hepatic cirrhosis: successful treatment by transjugular intrahepatic portosystemic shunt. J Thorac Imaging 17:233-236

4. Rosenberger A, Abrams HL (1971) Radiology of the thoracic duct. AJR $111: 807-820$ 
5. Mannella P, Cinotti A, Soriani M, et al. (1980) Radiology of the thoracic duct in liver cirrhosis. Radiol Med (Torino) 66:243-245

6. Zironi G, Cavalli G, Casali A, et al. (1995) Sonographic assessment of the distal end of the thoracic duct in healthy volunteers and in patients with portal hypertension. AJR 165:863-866

7. Schieber W (1965) Lymphangiographic demonstration of thoracic duct dilation in portal cirrhosis. Surgery 57:522-524

8. Verma SK, Mitchell DG, Bergin D, et al. (2007) The cisterna chyli: enhancement on delayed MR images after intravenous administration of gadolinium chelate. Radiology 24:776-783

9. Child GC, Turcotte TG (1964) Surgery, portal hypertension. In: Child CG (ed) The Liver and Portal Hypertension. Philadelphia: Cirrhosis. WB Saunders, pp 50

10. Infante RC, Esnaola S, Villeneuve JP (1987) Clinical and statistical validity of conventiona prognostic factors in predicting short-term survival among cirrhotics. Hepatology 7:660-664

11. Thomsen C, Becker U, Winkler K, et al. (1994) Quantification of liver fat using magnetic resonance spectroscopy. Magn Reson Imaging 12:487-495

12. Takahashi H, Kuboyama S, Abe H, et al. (2003) Clinical feasibility of noncontrast-enhanced magnetic resonance lymphography of the thoracic duct. Chest 124:2136-2142

13. Schwartz M (1961) A biomathematical approach to clinical tumor growth. Cancer 14:1272-1294 
14. Shrout PE, Fleiss JL (1979) Intraclass correlations: uses in assessing rater reliability. Psychol Bull 86:420-429

15. Pinto PS, Sirlin CB, Andrade-Barreto OA (2004) Cisterna chyli at routine abdominal MR imaging: a normal anatomic structure in the retrocrural space. Radiographics 24:809-817

16. Tamsel S, Ozbek SS, Sever A, et al. (2006) Unusually large cisterna chyli: US and MRI findings. Abdom Imaging 31:719-721

17. Smith T, Grigoropoulos J (2000) The cisterna chyli: incidence and characteristics on CT. Clin Imaging 26:18-22

18. Erden A, Fitoz S, Yagmurlu B, et al. (2005) Abdominal confluence of lymph trunks: detectability and morphology on heavily T2weighted images. AJR 184:35-40

19. Dumont AE, Mulholland JH (1960) Flow rate and composition of thoracic duct lymph in patients with cirrhosis. N Engl J Med 263:471-474

20. Elk JR, Laine GA (2000) Pressure within the thoracic duct modulates lymph composition. Microvasc Res 39:315-321

21. Witte CL, Witte MH, Dumont AE, et al. (1968) Lymph protein in hepatic cirrhosis and experimental hepatic and portal venous hypertension. Ann Surg 168:567-577

22. Nazyrov FG, Khoroshaev VA, Deviatov AV, et al. (1989) Characteristics of portal-lymphatic hypertension and surgical treatment of patients with cirrhosis of the liver and persistent ascites. Vestn Khir Im I I Grek 142:104-106 23. Aspestrand F, Schrumpf E, Jacobsen M, et al. (1991) Increased lymphatic 
flow from the liver in different intra and extrahepatic diseases demonstrated by CT. J Comput Assist Tomogr 15:550-554

24. Mitchell DG, Lovett KE, Hann HWL, et al. (1993) Cirrhosis: multiobserver analysis of hepatic MR imaging findings in a heterogeneous population. JMRI 3:313-321

25. Ito K, Mitchell DG, Hann HWL, et al. (1998) Progressive viral-induced cirrhosis: serial MR imaging findings and clinical correlation. Radiology 207:729-735

\section{FIGURES \& TABLES}

Table 1. MRI visualization of cisterna chyli (CC) in 149 patients with compensated and uncompensated cirrhosis.

Findings Compensated Uncompensated P-value

$(n=43)(n=106)$

$\mathrm{CC}>2 \mathrm{~mm} 2588<0.001$

CC $>6 \mathrm{~mm}$ (dilated) $257<0.001$

Note: Data are the number of patients 
Fig. 1. MR images of cisterna chyli (CC) in a 56-year-old man with uncompensated cirrhosis. (A) Coronal single-shot FSE T2-weighted MR image (TE 180-200 ms) shows dilated (8 $\mathrm{mm}$ in diameter) hyperintense CC (arrow) in the right retrocrural space. (B) Axial 3D gradient-echo delayed phase MR image (5/1.4, 12flip angle) demonstrates dilated CC (8 $\mathrm{mm}$ in diameter) (arrowhead) enhancing intensely similar to surrounding blood vessels. Signs of portal hypertension splenomegaly and patent paraumblical shunt (arrow) are evident. 


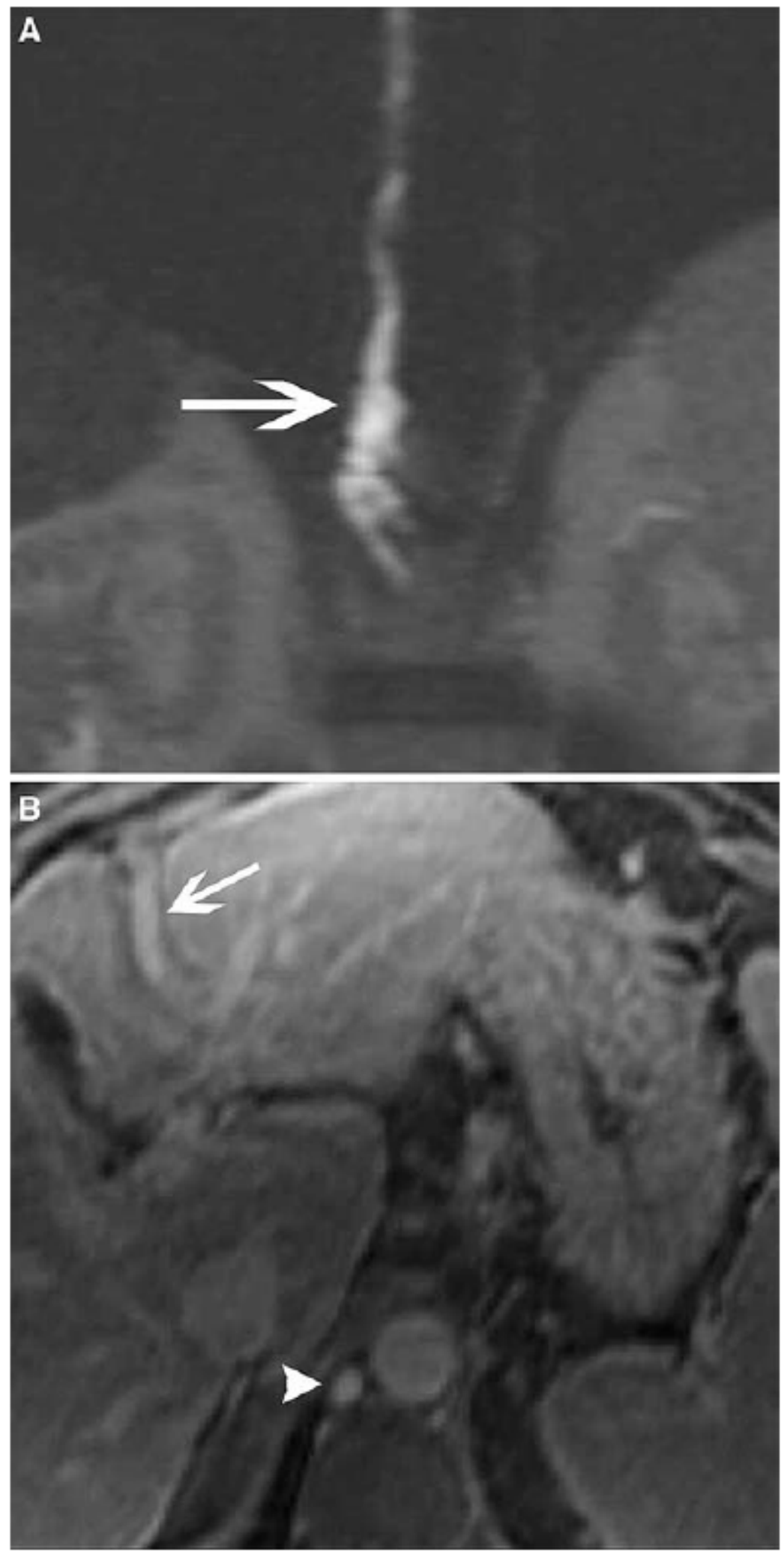


Fig. 2. MR images of cisterna chyli (CC) in a 48-year-old man with compensated cirrhosis and right-sided cardiac decompensation. (A) Axial single-shot FSE T2weighted MR image (TE 180-200 ms) shows dilated (11 mm in diameter) hyperintense CC (arrow) in the right retrocrural space. (B) Axial 3D gradient-echo portal venous phase MR image $(5 / 1.4,12 f$ lip angle) shows dilated hepatic veins and prominent IVC (arrow), consistent with right-sided heart failure/tricuspid regurgitation. 

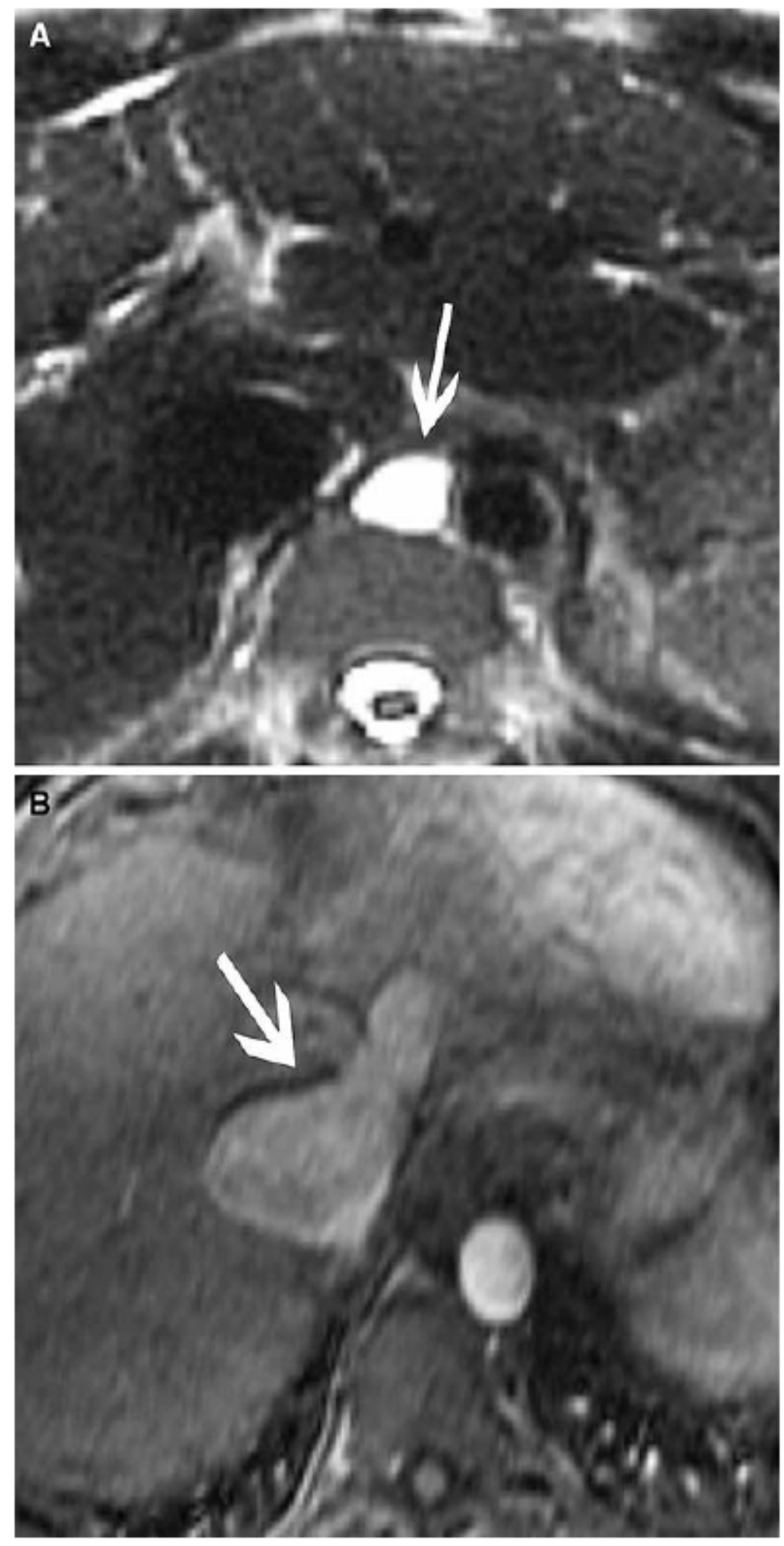
Fig. 3. MR images of cisterna chyli (CC) in a 47-year-old man with cirrhosis with clinical progression. (A) Axial single-shot FSE T2-weighted MR image (TE 180-200 $\mathrm{ms}$ ) shows minimal ascites (arrow) and hyperintense (4 $\mathrm{mm}$ in diameter) CC (arrowhead). (B) Axial single-shot FSE T2-weighted MR image (TE 180-200 ms) obtained 9 months later shows moderate ascites (arrow) and hyperintense CC with diameter (5 $\mathrm{mm})$ (arrowhead).

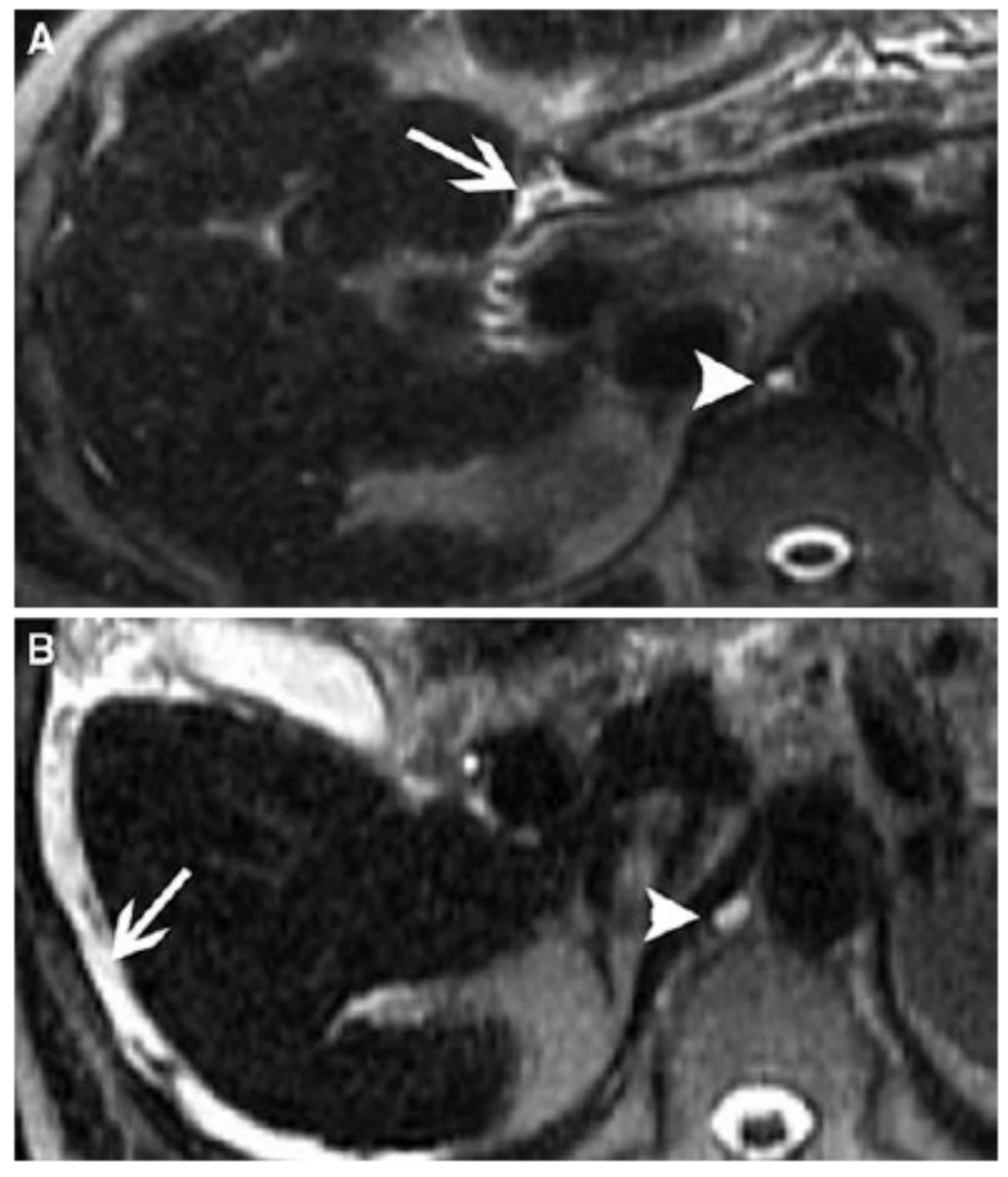

